\title{
Electron Histochemical Studies of Acid phosphatase Activity in Rat Endometrium.
}

\author{
Kaz'o Ogawa, Yasuyuki Shinonaga and Yasuko Seito, \\ Department of Anatony, Kyoto University School of Medicine
}

The electron histochemical detection of acid P-ase was done after Ogawa's method. Acid P-ase activity was positive in the cytoplasmic membrane of microvilli of epithelial cells of endometrium and in lysosomes scattered in cytoplasm.

Endoplasmic reticulum of phagocytes in stroma was also positive. Nonspecific depositions of lead on nuclei were occasionally recognized. Though acid phosphatase activity was occasionally positive in all cytoplasmic components, including microvilli, mitochondria and endoplasmic reticulum, of some epithelial cells of endometrium, the significance of this findings is not clear.

\section{Intracellular Localization of Phosphorylase from Histochemical and Electron-microscopic Observations.}

\author{
Mitsuo SASAKI and Tadao TAKeUchI \\ Department of Pathology, Kumamoto University School of Medicine, Kumamoto.
}

Polysaccharide synthesized histochemically from glucose-1-phosphate by the phosphorylase activity, with a new method of preparing ultrathin sections for electron microscope, was observed in skeletal muscles. For incubation was

Fig. 1-2 Low-density, amorphous cloudy substances (P) showing polysaccharide newly synthesized from glucose-1-phosphate by phosphorylase are noted in the sarcoplasmic matrix among the myofibriles (Mf) and other organelles. This polysaccharide stains blue with iodine in the histochemical procedure of $1 \mu$ section cut from the same block for light microscopic use. Mitochondria (M) are disturbed by incubating in the substrate medium, but the double membrane can be identified (Osmium-methachrylate ultrathin section without staining).

Fig. 3 Synthesized polysaccharide $(P)$ is seen by staining with Watson's lead method. It is difficult to stain, but some of the stainable particles are distributed under the cell membrane $(\mathrm{Cm})$. 